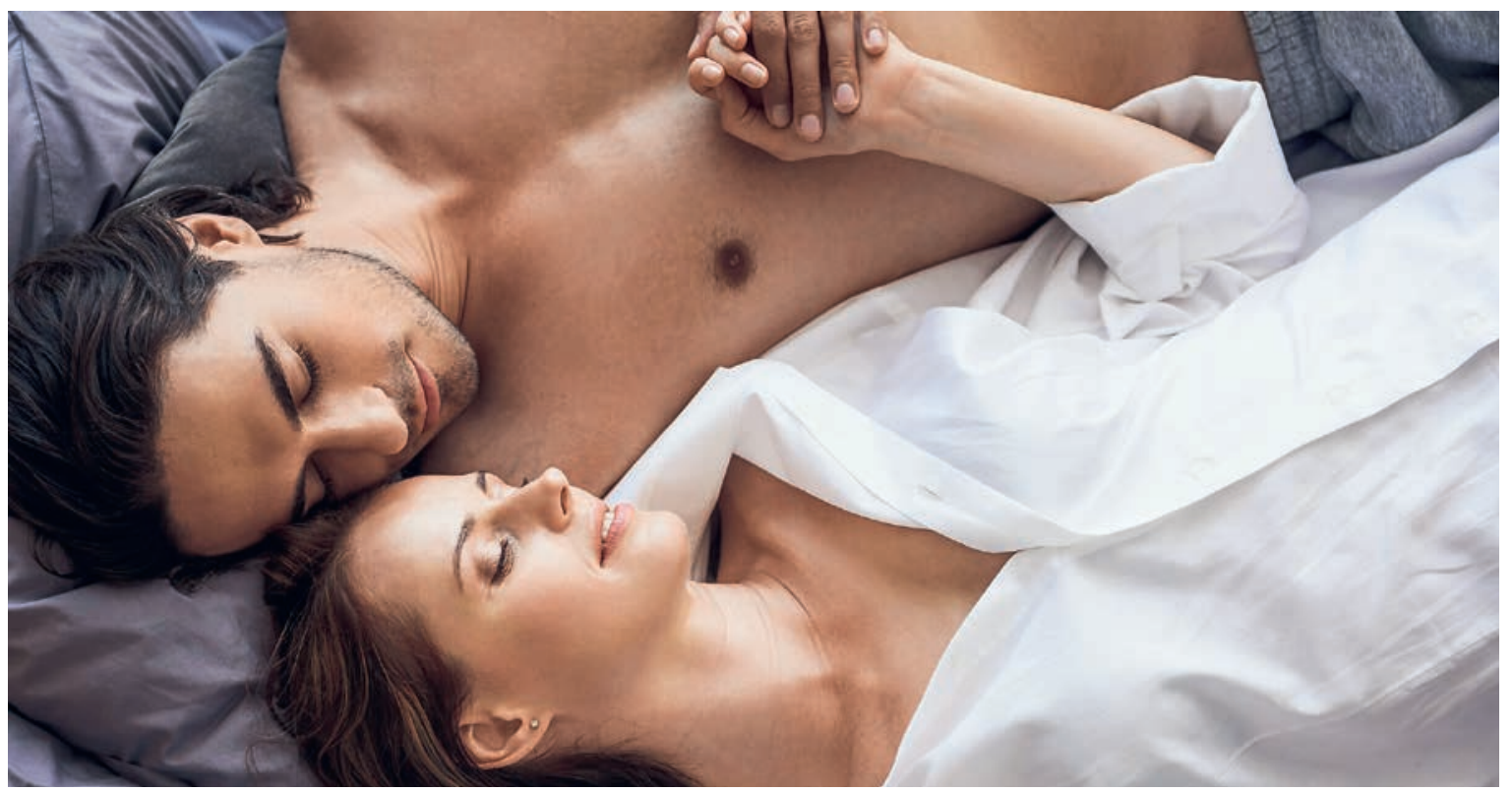

\title{
Beratungsstellen für sexuelle Gesundheit: Zu Ihren Diensten!
}

\section{Alain Pfammattera, Cate Esson ${ }^{b}$}

a Verantwortlicher Leiter des Fachbereichs Beratung, Fachperson sexuelle Gesundheit der Beratungsstelle sexuelle Gesundheit - Familienplanung der Stiftung PROFA, Kanton Waadt. Diplom of Advanced Studies DAS in sexueller Gesundheit; ${ }^{b}$ Stellvertretende ärztliche Leiterin der Beratungsstelle sexuelle Gesundheit - Familienplanung der Stiftung PROFA, Kanton Waadt, MBChB (Glasgow) MRCGP

1 A. Späth, C. Schneider, L. Stutz, S. Tschudin und E. Zemp Stutz (2017) Schweizerischer Verhütungsbericht (Obsan-Dossier 59). Neuenburg: Schweizerisches Gesundheitsobservatorium (Obsan)

Gemäss dem Schweizerischen Verhütungsbericht ${ }^{1}$ nimmt jede vierte Frau unter 50 Jahren die Pille. In den letzten Jahren rücken andere Methoden in den Vordergrund, und die Zahl der Frauen, die eine hormonfreie Verhütung bevorzugen, steigt. Behandelnde Ärztinnen und Ärzte wie auch Beratungsstellen für sexuelle Gesundheit sind mit diesem neuen Trend konfrontiert. Um sicherzustellen, dass jede Person ihre Sexualität leben kann, ohne eine ungeplante Schwangerschaft zu riskieren, gilt es eine informierte Wahl zu ermöglichen und Frauen und ihre Partner über das gesamte Spektrum der Verhütungsmethoden zu informieren.

\section{Beratungsstellen für sexuelle Gesundheit}

Im Zeitalter des Internets zirkulieren zahlreiche Informationen über Verhütung. Manche sind korrekt, andere nicht. Patientinnen gelangen mit vielen Fragen an ihre behandelnde Ärztin bzw. ihren behandelnden Arzt. Es braucht Zeit, diese Fragen zu beantworten und so die Wahl eines geeigneten Verhütungsmittels zu ermöglichen. Ärztinnen und Ärzte haben die Möglichkeit, ihre Patientinnen an eine Beratungsstelle für

\section{SEXUELLE GESUNDHEIT Schweiz}

ist der Dachverband der Beratungsstellen, Fachorganisationen und Fachpersonen, die im Bereich der sexuellen und reproduktiven Gesundheit und der Sexualaufklärung in der Schweiz tätig sind. Sie ist Partnerin des Bundesamtes für Gesundheit bei der Umsetzung des Nationalen Programms HIV und andere sexuell übertragbare Infektionen (NPHS). SEXUELLE GESUNDHEIT Schweiz engagiert sich auf nationaler sowie auf internationaler Ebene für eine umfassende Sexualaufklärung und die Promotion und Einhaltung der sexuellen Rechte. SEXUELLE GESUNDHEIT Schweiz ist akkreditiertes Mitglied der International Planned Parenthood Federation (IPPF) 


\begin{tabular}{|c|c|c|c|}
\hline $\begin{array}{l}\text { spermizidem Gel } \\
\text { Portiokappe mit } \\
\text { spermizidem Gel }\end{array}$ & $\begin{array}{l}\text { Kupferball } \\
\text { Gynefix }^{\circledR}\end{array}$ & & - Sterilisation der Frau \\
\hline
\end{tabular}

sexuelle Gesundheit zu verweisen. Die Beratungsstellen bieten meist kostenlose Informationen und Beratung durch Fachpersonen der sexuellen Gesundheit zur Empfängnisverhütung und weiteren Themen an. Sie sind in ihrer Region eng mit weiteren Fachstellen vernetzt. Für behandelnde Ärztinnen und Ärzte sind diese Stellen damit eine wertvolle Ressource. Beispielsweise können Patientinnen zur kostenlosen Beratung überwiesen werden, bevor die Ärztin oder der Arzt eine Behandlung vornimmt oder ein Rezept ausstellt. Die gute Zusammenarbeit zwischen behandelnden Ärztinnen bzw. Ärzten und den Beratungsstellen als eine Art Tandem kann eine optimale Versorgung der Patientinnen gewährleisten.

\section{Im Auftrag der Kantone}

Basierend auf dem Bundesgesetz über die Schwangerschaftsberatungsstellen haben die Kantone Beratungsstellen mandatiert, um eine kostenlose, vertrauliche und ergebnisoffene Beratung zu gewährleisten (siehe Kasten 3). Die Beraterinnen und Berater sind auf dem Gebiet der sexuellen Gesundheit qualifiziert. Rund die Hälfte der Stellen verfügen zusätzlich über medizinisches Personal, sei dies innerhalb oder ausserhalb der Stelle. Diese interdisziplinäre Zusammenarbeit bietet eine wertvolle Ergänzung in Bezug auf die Empfängnisverhütung. Ratsuchende Einzelpersonen oder Paare werden über die ganze Bandbreite der Verhütungsmethoden informiert und durch die Fachpersonen

\section{Beratungsstellen für sexuelle Gesund- heit und Familienplanung}

In der Schweiz gibt es in allen Kantonen Beratungsstellen für sexuelle Gesundheit und Familienplanung (insgesamt 75). Diese gründen auf dem Bundesgesetz SR 857.5 aus dem Jahr 1981. Die Beratungsstellen bieten kostenlose, vertrauliche und individuelle Beratung an. Als Dachverband der Beratungsstellen leistet SEXUELLE GESUNDHEIT Schweiz einen wichtigen Beitrag zur Qualitätssicherung.

www.sexuelle-gesundheit.ch/schwangerschaftsberatungsstellen dabei unterstützt, Vor- und Nachteile abzuwägen und bewusst die für sie passende Wahl zu treffen. Für die medizinische Anamnese, das Ausschliessen von Kontraindikatoren oder zur Verschreibung von rezeptpflichtigen Mitteln findet anschliessend eine Überweisung an die Ärztin oder den Arzt statt, oder die Betroffenen vereinbaren selbständig einen Termin. Mit neuen Lebensumständen oder im Laufe der Zeit verändern sich oft die Ansprüche einer Frau oder eines Paares an die Empfängnisverhütung. Es tauchen vielleicht Fragen und Zweifel an der bisherigen Verhütungsmethode auf. In diesem Fall haben behandelnde Ärztinnen und Ärzte die Möglichkeit, ihre Patientinnen an die Fachpersonen für sexuelle Gesundheit zu überweisen, um Unsicherheiten und neue Methoden zu diskutieren.

Diese Zusammenarbeit zwischen Ärztinnen und Ärzten und den Beratungsstellen für sexuelle Gesundheit ermöglicht eine optimale Betreuung der Patientinnen und ihren Partnern.

\section{«Ich möchte natürlich verhüten!»}

Immer häufiger suchen Frauen nach Alternativen zur hormonellen Verhütung. Sie äussern den Wunsch, auf Hormone zu verzichten, da sie diese über lange Zeit eingenommen haben oder sie nicht mehr vertragen. Die spezialisierten Beratungsstellen für sexuelle Gesundheit verfügen über das Wissen, die Erfahrung und die Zeit, um die verschiedenen Optionen (siehe Kasten 2) zu besprechen. Ängsten oder falschen Überzeugungen kann mit stichhaltigen Informationen begegnet werden, so dass die Frau oder das Paar eine informierte Entscheidung treffen kann. Manchen Frauen wird durch das Beratungsgespräch bewusst, dass ihre bisherige Verhütungsmethode doch am besten zur aktuellen Situation passt. Andere entscheiden sich für eine neue Methode, die ihren Erwartungen besser entspricht. In Bezug auf die Compliance ist es wichtig, dass die Frau ihre Entscheidung basierend auf umfassender Beratung fällen kann. 


\section{Fünf Gründe für eine Zusammenarbeit}

\section{Ausgebildete Fachpersonen}

Die meisten Beraterinnen und Berater besitzen ein DAS (Diploma of Advanced Studies in der Westschweiz) oder einen MAS (Master of Advanced Studies in der Deutschschweiz) in sexueller Gesundheit. Sie sind qualifiziert, zu Empfängnisverhütung zu informieren und zu beraten. Ihr Fachgebiet deckt alle Aspekte der sexuellen und reproduktiven Gesundheit ab und ermöglicht eine ganzheitliche Beratung.

\section{Zeitliche Ressourcen}

Die Beratungen zu sexueller Gesundheit werden öffentlich finanziert und stehen der gesamten Bevölkerung offen. Im Bereich der Empfängnisverhütung können sämtliche hormonellen und nicht-hormonellen Methoden erklärt werden, so dass individuell diejenige gewählt werden kann, die sich in der jeweiligen Lebenssituation am besten eignet.

\section{Individuelle Beratung}

Grundsätzlich ist jeder Mensch in der Beratung willkommen. Die Fachpersonen legen besonders Wert auf die sorgfältige Beratung von Jugendlichen, Menschen in sozialen oder finanziellen Notlagen sowie Menschen mit Migrationserfahrung.

\section{Kompetentes medizinisches Personal}

Die Hälfte der Beratungsstellen für sexuelle Gesundheit sind medizinisch ausgerichtet und bieten die Dienste von Gynäkologinnen sowie Allgemeinmedizi-

\section{Sex-i.ch in elf Sprachen}

Für Ihre fremdsprachigen Patientinnen und Patienten: Diese spezielle Website von SEXUELLE GESUNDHEIT Schweiz informiert über alle Verhütungsmethoden, Schwangerschaft, Schwangerschaftsabbruch, Anatomie der Geschlechtsorgane von Frau und Mann sowie sexuell übertragbare Infektionen. In Zusammenarbeit mit Fachpersonen aus den Beratungsstellen werden die Informationen regelmässig aktualisiert oder neu entwickelt, wie zum Beispiel die aktuelle Ergänzung zu «hormonfreien Verhütungsmethoden». Die Informationsblätter können als PDF-Dateien in allen elf Sprachen an Patientinnen und Patienten abgegeben werden. www.sex-i.ch

nern mit breiter Erfahrung auf dem Gebiet der sexuellen Gesundheit an. Diese medizinischen Fachpersonen stehen ihren niedergelassenen Kolleginnen und Kollegen für spezifische Auskünfte zur Verfügung. Das Team der ärztlich dotierten Beratungsstellen für sexuelle Gesundheit umfasst auch medizinische Praxisassistentinnen, die über grosse Erfahrung in diesem Bereich verfügen.

\section{Zusammenarbeit mit Dolmetschenden}

Damit jede Person mit ihren Anliegen verstanden und angemessen informiert werden kann, können die Stellen bei der Beratung von Fremdsprachigen professionelle Dolmetschende einsetzen. In verschiedenen Kantonen sind diese Dolmetschenden speziell in Fragen der sexuellen Gesundheit geschult.

Bildnachweis

(c) Iakovenko123 | Dreamstime.com 